\title{
Evaluation on the Status Quo of Mass Sporting Activities in the Urban Area of Weinan City
}

\author{
Li Chunlan* \\ College of Education and Public Management, Weinan Normal University, Shannxi 714099
}

\begin{abstract}
By applying the methods of questionnaire survey, field investigation, literature data, this paper investigates the implementation of the mass sporting program in the urban area of Weinan, and by referring to related data from the Sports Bureau of Weinan city, this paper conducts an analysis on the investigation result based on Evaluation Criteria for the Implementation of the "National Fitness Program (2011-2015)". It is found that, in the urban area of Weinan city, both the per capita area of sports venues and the number of sports activity sites have almost reached the national standard B and there are plenty of people who regularly participate in sports activities, but social sports instructors are obviously insufficient. Based on the analysis, this paper puts forward suggestions to promote the development of mass sporting program in a faster and better way in this city.
\end{abstract}

KEYWORD: urban area of Weinan city; mass sporting (National Fitness Program); standards; evaluation

\section{INTRODUCTION}

After the first and second phase of the implementation of the "National Fitness Program", the people's awareness on the importance of participation of sports activities and its pervasiveness have been greatly enhanced and the number of sporting facilities and venues have increased obviously. With the gradual development of mass sporting activities, people's life concept has also been changed. To further and more widely develop mass sporting cause and continue to improve the people's health, a new plan of the "National Fitness Program 2011-2015" has been issued, which has raised new requirements on the aims and tasks of national fitness program. In 2007, the government of Shannxi Province has also passed and implemented the "Shannxi Provincial Mass Sporting Regulations" according to the characteristics of the mass sporting activities. To have a better knowledge of the mass sporting activities in Weinan city since the implementation of the regulations and evaluate whether this situation has met the standards of the regulations, our research team has reached an objective conclusion and put forward feasible suggestions according to the analysis of the results from the investigation of the implementation of the mass sporting activities in the urban area of Weinan city, in order to provide theoretical basis for the better development and implementation of the mass sporting program in the city.

\section{RESEARCH OBJECTS AND METHODS}

The research objects of this paper are community residents in the urban area of Weinan city, including Linwei District, Gaoxin District, and Jingkai District. By applying the methods of questionnaire survey, field investigation, literature data, and mathematical statistics, and on the basis of the related data from the Sports Bureau of Weinan city, this paper has conducted the study and analysis on the sports venues areas, sports sites for morning and evening exercises, population groups who often take part in activities, social sports instructors and so on in the urban area of the city. A total of 500 questionnaires have been sent out and then collected, with $98 \%$ of them valid.

\section{INVESTIGATION RESULTS AND ANALYSIS}

The urban area of Weinan city includes Linwei District, Gaoxin District, and Jingkai District, and the area has a population of 0.4 million. There are 10 subdistrict offices in these four districts: Renmin

\footnotetext{
* Author: Li Chunlan (1975-), Associate Professor, College of Education and Public Management Research Interests: Sport Sociology.
} 
subdistrict office, Xiangyang subdistrict office, Duqiao subdistrict office, Jiefang subdistrict office, Zhan'nan subdistrict office, Shuangwang subdistrict office, Liangtian subdistrict office, Baiyang subdistrict office, Chongye subdistrict office, and New City Government office.

\subsection{The area of various sports venues in the urban area of Weinan city}

According to the data from the census about sports venues around the country in 2003, China has 850 thousand sports venues and the per capita area of sports venues (total area of sports venues/the number of population) is $1.03 \mathrm{~m}^{2}$. By the end of 2010 , the number of sports venues in China has exceeded 1 million and the per capita area of sports venues was $1.14 \mathrm{~m}^{2}$, which indicates that both the number and area of sports facilities in China have made great progress. As shown in the Table 1, the total area of sports venues in the urban area of Weinan city is $583,107 \mathrm{~m} 2$ and the per capita area of sports venues is $1.46 \mathrm{~m}^{2}$. According to the standards of three classes of per capita area of sports venues, which is defined in the "National Fitness Program 20112015 ", the standard C for per capita area of sports venues is over $1.0 \mathrm{~m}^{2}$, the standard $\mathrm{B}$ is over $1.5 \mathrm{~m}^{2}$, and the standard $\mathrm{A}$ is more than $1.8 \mathrm{~m}^{2}$. It is observed that, the current per capita area of sports venues in Weinan city has reached the standard C, approaching the standard B. However, to reach the standard A, the city government still needs to continue to increase the investment on the construction of public sports facilities at the subdistrict level in the coming year and effectively implement the tasks as set in the "Plan for the Construction of Basic Public Service Sports Facilities" in the " $12^{\text {th }}$ Five-year Plan".

Table 1. The areas of various sports venues $\left(\mathrm{m}^{2}\right)$

\begin{tabular}{|l|l|l|l|l|l|l|}
\hline & Public venues & Private venues & Venues at schools & Venues in enterprises & Venues in communities & Total \\
\hline Linwei District & 98944 & 8550 & 144830 & 19600 & 7793 & 279717 \\
\hline Gaoxin District & 136337 & $/$ & 95539 & 10700 & 50 & 242626 \\
\hline Jingkai District & 7120 & $/$ & 53024 & 620 & $/$ & 60764 \\
\hline Total & 242401 & 8550 & 293393 & 30920 & 7843 & 583107 \\
\hline
\end{tabular}

\subsection{The sporting activity sites of morning and evening exercise in the urban area of Weinan city}

Sports activity sites for morning and evening exercise are the most typical activity sites in urban communities. Majority of this type of sites are managed by residents themselves (about 75.8\%), while only few of them are organized by subdistrict or communities offices, sports associations, trade unions of organizations in areas, and management offices for the retirees, accounting for no more than $5 \%$, which is an important characteristic of the management and organization of mass sports activities in subdistricts and communities in China. It can be seen from Table 2 and the data from the Sports Bureau of Weinan city, there are 63 sports activity sites for morning and evening exercise in the urban area of Weinan city in total, 50 sports activity sites in other forms, 40 sites for fitness, and 25 sports venues in communities. More than 6,132 people regularly participate in sporting activities in these sites. According to the classification of sports sites defined in the "National Fitness Program 2011-2015", 2 sites for every 10 thousand residents meets the standard C, 3 sites for every 10 thousand residents meets the standard $\mathrm{B}$ and, 5 sites for every 10 thousand residents meets the standard A. It can be seen that, there are 2.8 sports sites for every 10 thousand residents in the urban area of Weinan city and the sites' coverage is $40 \%$, which has reached the minimum requirement of National Fitness Program Evaluation Criteria, and is gradually approaching to Standard B.

Table 2. Summary of Morning and Evening Exercises

\begin{tabular}{|c|c|c|c|}
\hline & Number of sites & Number of Participants (person) & Number of sites with sports facilities \\
\hline Renmin subdistrict office & 11 & 570 & 4 \\
\hline Xiangyang subdistrict office & 6 & 422 & 4 \\
\hline Jiefang subdistrict office & 8 & 950 & 5 \\
\hline Duqiao subdistrict office & 5 & 850 & 4 \\
\hline Zhan'nan subdistrict office & 8 & 1020 & 5 \\
\hline Shuangwang subdistrict office & 5 & 230 & 3 \\
\hline Liangtian subdistrict office & 5 & 765 & 4 \\
\hline Baiyang subdistrict office & 5 & 490 & 4 \\
\hline Chongye subdistrict office & 5 & 270 & 4 \\
\hline New City Government office & 5 & 565 & 3 \\
\hline Total & 63 & 6132 & 40 \\
\hline
\end{tabular}




\subsection{Participants of mass sporting activities in the urban area of Weinan city}

It can be seen from the results of the investigation, the proportion of regular participants of sports activities in the urban area of Weinan city is about $33 \%$, the proportion of occasional participants is about $47 \%$, and the proportion of people who have intention to participate in the sports activities in the future is about $2 \%$. The proportion of the regular participants of sports activities refers to the proportion of those residents who take exercise more than three times each week, over half an hour each time and the intensity of whose exercise is higher than the moderate level, to the total population of the area. When calculating the proportion of regular participants of sporting activities with their age over 16 in urban area, school students are not included. Based on the "National Fitness Program 20112015 ", the participation rate of sports activities in the urban area of Weinan city has reached over 32\%, which meets the standard B. If those who are willing to participate in the sports activities in the future in the urban area of Weinan city can be quickly taken in, this rate will further increase in the future. Based on Table 1, it is found that, although the number of sports sites in the urban area of Weinan city only reached standard $\mathrm{C}$, the number of participants has reached standard $B$, which proves that the participation rate of sports activities of residents in this city is comparatively high and the influence of mass sporting activities is great among these residents. 3.4 Social sports instructors in the urban area of Weinan city

Table 3 Statistics of Participants of Mass Sporting Activities (Person)

\begin{tabular}{|c|c|c|c|c|}
\hline & Regular participants & never & willing to & total \\
\hline & Proportion & Proportion & Proportion & \\
\hline $16-35$ & $16 \%$ & $18 \%$ & $0.40 \%$ & 200 \\
\hline $36-55$ & $11 \%$ & $21 \%$ & $1 \%$ & 207 \\
\hline over 56 & $6 \%$ & $8 \%$ & $0.60 \%$ & 93 \\
\hline Total & $33 \%$ & $47 \%$ & $2 \%$ & \\
\hline
\end{tabular}

Social sports instructors are those voluntary instructors for social sports cause, and the total number, working enthusiasm and personal capability of the social sports instructors may affect the effects of their instruction. There are 1,300 social sports instructors in the urban area of Weinan city and they are from state organs, schools, sports departments, activity sites and sports clubs. According to the assessment criteria of the "National Fitness Program 2011-2015", Standard C requires that the number of social sports instructors who have got the technical grading certificates should reach $0.5 \%$ of the total population, standard $\mathrm{B}$ requires to reach $1 \%$, and Standard A requires to reach $1.5 \%$. The proportion of social sports instructors is $0.3 \%$ in Weinan city, which only meets the goal of Shannxi province, but has not reached the standard $\mathrm{C}$ of the "National Fitness Program 2011-2015". It can be seen that the Sports Bureau of Weinan city has to intensify trainings for social sports instructors, so as to largely raise the number of social sports instructors.

\section{CONCLUSION AND SUGGESTIONS}

\subsection{Conclusion}

The per capita area of sports venues and the number of sports activity sites in the urban area of Weinan city have both reached the standard $\mathrm{C}$ of the

"National Fitness Program 2011-2015", approaching the standard $\mathrm{B}$.

There are plenty of people who regularly participate in sports activities in the urban area of Weinan city, especially the huge number of people doing morning and evening exercise. According to the "National Fitness Program 2011-2015", the participation rate of sports activities in this area has reached the standard $\mathrm{B}$.

The social sports instructors in this area is obviously insufficient and the number of social sports instructors has not reached the minimum requirement of the "National Fitness Program 2011$2015^{\prime \prime}$.

\subsection{Suggestions}

On the basis of implementation of the "National Fitness Program 2011-2015" and various mass sporting regulations of Shannxi province, the government of Weinan city should continue to invest in the construction of sports infrastructure, organize and develop more sports activity sites and encourage more residents to take part in sports activities. The Sports Bureau of Weinan city should intensify the trainings on social sports instructors in the various ways, such as regular training, structured training for different levels, and the experienced to train the inexperienced. Then they can quickly become the social sports instructors and provide services for the mass sporting cause. The government of Weinan city should follow the evaluation criterion for implementation of the "National Fitness Program 2011-2015" and use the criterion to promote the construction, development, and effects of the mass sporting program, to truly put it into practice.

\section{ACKNOWLEDGEMENT}

The program of Education Bureau of Shannxi Province (2013JK0517) 


\section{REFERENCES}

[1] Wang Kai Zhen, Zhao Li, Community Sports. Beijing: Higher Education Press, 2004

[2] Wang Kai Zhen, Li Xiangru, Community Sports Instruction. Guangxi: Guangxi Normal University Press, 2005

[3] Evaluation Criterion [EB/OL] for Implementation of the "National Fitness Program 2011-2015". http://www.sport.gov.cn/n16/n33193/n33208/n33418/n33 598/2046496.html, August 26th 2011.

[4] Reseach team of China's Mass Sporting Activities, Investigation and Research on the Status Quo of China's
Mass Sporting Activities. Beijing Sports University Press, 2005.

[5] "National Fitness Program 2011-2015" [EB/OL]. http://www.sport.gov.cn/n16/n1077/n1467/n1808585/180 8663.html, February 24th, 2011.

[6] Liuhong Zang Norm analysis of physical shape on Kazak nationality in XinJiang, Proceedings of the 2010 international symposium on children and youth fitness and health, 2010.10.

[7] Liuhong Zang. A Study on Application of Dummy Reality Technology in Development of Xinjiang Minority Nationality Gymnasium Tour, Proceedings of second international conference on sports science and sports engineering. 2009.9. 